\title{
Binding across space and time in visual working memory
}

\author{
Paul Johan Karlsen \\ University of Tromsø, Tromsø, Norway \\ Richard J. Allen \\ University of Leeds, Leeds, England \\ AND \\ Alan D. Baddeley and Graham J. Hitch \\ University of York, York, England
}

\begin{abstract}
Recent studies of visual short-term memory have suggested that the binding of features such as color and shape into remembered objects is relatively automatic. A series of seven experiments broadened this investigation by comparing the immediate retention of colored shapes with performance when color and shape were separated either spatially or temporally, with participants required actively to form the bound object. Attentional load was manipulated with a demanding concurrent task, and retention in working memory was then tested using a single recognition probe. Both spatial and temporal separation of features tended to impair performance, as did the concurrent task. There was, however, no evidence for greater attentional disruption of performance as a result of either spatial or temporal separation of features. Implications for the process of binding in visual working memory are discussed, and an interpretation is offered in terms of the episodic buffer component of working memory, which is assumed to be a passive store capable of holding bound objects, but not of performing the binding.
\end{abstract}

Perceiving the world involves simultaneously processing across a range of channels of separable features, such as shape, color, and location, followed by a process in which they are bound together into objects, scenes, and episodes. The subsequent binding processes and the temporary storage involved have formed a major focus for recent work in the area of visual short-term and working memory (e.g., Allen, Baddeley, \& Hitch, 2006; Fougnie \& Marois, 2009; Vogel, Woodman, \& Luck, 2001; Wheeler \& Treisman, 2002). A central question has been the extent to which this binding process is attention demanding. This has been tackled in three ways. The first approach has been to compare the accuracy of immediate recognition memory for bound features, such as shape and color, with recognition of the constituent individual features. In general, any deficit attributable to binding has been small, suggesting a relatively automatic process (e.g., Vogel et al., 2001). A second approach concerns the retention of bound versus individual features over brief delays. Results here suggest that the bindings may be relatively fragile when the interval contains other visual activities, such as encoding or scanning an array or sequence of subsequent items (Allen et al., 2006; Alvarez \& Thompson, 2009; Fougnie \& Marois, 2009; Logie, Brockmole, \& Vandenbroucke, 2009; Shafritz, Gore, \& Marois, 2002; Wheeler \& Treisman,
2002). A third approach uses the concurrent task procedure to investigate whether attention-demanding activities differentially interfere with the binding process. Again, the evidence appears to suggest that binding is relatively automatic; although overall retention performance is affected by the concurrent attentional tasks, the effect is no greater for bound than for separate features (Allen et al., 2006; Cowan, Naveh-Benjamin, Kilb, \& Saults, 2006).

One way of conceptualizing the process of binding features into objects and chunks was proposed by Baddeley (2000) through the addition of a fourth component, the episodic buffer, to the multicomponent working memory model first proposed by Baddeley and Hitch (1974). The episodic buffer is assumed to comprise a limited-capacity multidimensional store that provides a link between the subcomponents of working memory, perception, and long-term memory and is assumed to be accessible through conscious awareness (Baddeley, 2000, 2007). In its initial formulation, it was suggested that information from perception and the visuospatial and verbal short-term stores accessed the episodic buffer through the central executive. Direct links from the phonological loop and sketchpad were expressly excluded on the grounds of parsimony, with the assumption that disrupting the central executive and studying the impact on feature binding would test this empirically. 
Allen et al. (2006) used dual-task methodology to tackle this assumption, predicting that if the central executive were essential to feature binding, an attention-demanding concurrent task should differentially disrupt the binding of features, over and above any impact on retention performance based on individual features. No evidence of this interaction was observed. Woodman, Vecera, and Luck (2003) have found evidence for the grouping of features in memory on the basis of bottom-up Gestalt principles. In line with this, the symmetry advantage in immediate visuospatial recall observed by Rossi-Arnaud, Pieroni, and Baddeley (2006) did not interact with any of a range of concurrent tasks aimed at disrupting various components of working memory, suggesting that visual binding based on Gestalt principles is also independent of executive control.

It could be argued that the operation of Gestalt principles and the simple binding of features into objects occur at a preattentive stage that is simply too peripheral to involve working memory. However, it is likely that there are different mechanisms underpinning different types of binding, with varying involvement of one or more subsystems of working memory or long-term memory. Thus, initially one may want to distinguish between a set of relatively automatic bindings that occur instantly when sufficient attention is given to the processing of the individual features or elements and a more active attention-demanding process involving effortful binding and maintenance (Allen et al., 2006). The form of binding involved in a task may vary as a function of a combination of factors, including object complexity, configuration, and exposure duration. An important challenge, therefore, is to identify and delineate when different forms of binding process are invoked (see also Allen, Hitch, \& Baddeley, 2009).

It has been known for some time that there are unitization effects in visual memory, whereby feature conjunctions are remembered better when the features are perceived to belong to the same object than when they are not-for example, by presenting them at separate spatial locations (Asch, Ceraso, \& Heimer, 1960). Spatial unitization effects have consistently been observed in visual working memory (see, e.g., Delvenne \& Bruyer, 2004; Xu, 2002a, $2002 \mathrm{~b}$ ). Physical attributes such as salience and grouping affect both what comes together as integrated percepts and what is temporarily stored as integrated objects in working memory. In line with this, Holcombe and Cavanagh (2001) found that spatially superposed features could be bound after very brief exposures, with binding presumably occurring at early stages of perception. In contrast, the pairing of spatially separated features required slower presentation rates, suggesting that perceiving such pairs might require binding at a slow, attentionally limited stage. It is possible that feature bindings can develop automatically through perceptual processes only when the features are part of a visually unitized object or pattern. Separation of features across space or time may rule out automatic, perceptually based binding and may shift responsibility to an attention-demanding set of processes. We therefore carried out a series of experiments to explicitly explore this possibility.
It is important to clarify that the form of attention explored in this series of experiments is a general processing and control resource provided by the central executive component of working memory (e.g., Baddeley, 1996, 2000) and based within the prefrontal cortex (see Kane \& Engle, 2002; Stuss \& Knight, 2002). Although it is possible that the central executive can be fractionated into further subsystems (e.g., Baddeley, 2002; Miyake et al., 2000), as a general resource it does make an important contribution to visuospatial processing (Miyake, Friedman, Rettinger, Shah, \& Hegarty, 2001; Posner, Inhoff, Friedrich, \& Cohen, 1987). This form of attention contrasts with a more specialized, parietal-based spatial attention resource that also contributes to visual perception and memory tasks (e.g., Posner et al., 1987). Treisman (e.g., 1982; Treisman \& Gelade, 1980) has suggested that focused visuospatial attention is particularly important in initial feature binding, although evidence for its role in the subsequent maintenance of bound representations is somewhat mixed (e.g., Fougnie \& Marois, 2009; Gajewski \& Brockmole, 2006; Johnson, Hollingworth, \& Luck, 2008; Yeh, Yang, \& Chiu, 2005). Thus, we are concerned here with whether general executive attention is critical for binding together spatially or temporally separated feature pairings in working memory.

\section{Overview of the Present Study}

In the experiments that follow, we explored the conditions under which the binding of features occurs automatically or requires additional executive resources. Specifically, we examined binding when features were spatially separated or separated in time. A first aim of the present study was to establish whether it is possible for spatially (Experiments 1-4) or temporally (Experiments 5-7) separated features to be accurately bound together and subsequently retrieved in response to a memory probe. Some studies have indicated that spatially separated features can be bound in memory, although subsequent recognition performance is often less accurate than that for unitized binding (e.g., Delvenne \& Bruyer, 2004; Xu, 2002a).

Second, we used demanding tasks performed concurrently with encoding and retention to study the role of central attention (Experiments 2, 4, 6, and 7). If the initial Baddeley (2000) hypothesis is correct, these atypical forms of binding should be more demanding of central executive resources than those based on colocation or symmetry, making them particularly vulnerable to interference during the performance of an attention-absorbing concurrent task. Alternatively, even spatially and temporally separated features may be automatically bound and stored in working memory.

\section{METHOD}

The following procedure and materials were used in all the experiments, unless otherwise noted.

\section{Participants}

A different group of undergraduate students drawn from New York University and the University of Oslo took part in each ex- 
periment for course credit or financial reward. All had normal color vision as assessed by the Tabulæ Pseudoisochromaticæ B.-K. color blindness test (Boström \& Kugelberg, 1981).

\section{Materials, Design, and Procedure}

All the stimuli and probe items were simple shapes subtending a visual angle of approximately $0.95^{\circ}$, presented on a white background. There was a pool of eight colors (black, blue, green, gray, red, turquoise, violet, and yellow), all selected on the basis of ease of discriminability. There was a pool of eight shapes, along with one neutral shape. The latter was used in spatially separated, temporally separated, and color-only conditions, for the presentation of color. In the shape-only and in separated conditions, unfilled 3-point black outlines were used for the presentation of shape.

Examples of the unitized and spatially separated stimuli from Experiments 1-4 are displayed in Figure 1, using the presentation method from Experiments 3 and 4 as an illustrative example. Unitized feature combinations consisted of a color and a shape presented together in space, as a single-colored shape. Spatially separate combinations comprised a color and a shape presented simultaneously, but at vertically adjacent locations separated by a visual angle of approximately $0.38^{\circ}$. In the spatially separated feature condition, the shape elements were always presented directly above the colors, in the same location as the objects in the unitized condition. Temporally separate conditions are not illustrated but are described in detail in Experiments 5-7. They involved presenting a set of shapes on screen, followed by a brief delay and then a set of colors in the same locations. Features were not repeated within a trial for the majority of trials. A minority of trials in each condition did involve a repetition of color or shape, in order to rule out possible confounding response strategies (see Allen et al., 2006). These trials were discarded from further analysis.

Trials in all the conditions commenced with a fixation cross at screen center for $500 \mathrm{msec}$, followed by a 250 -msec blank screen delay and then the first of the to-be-remembered stimuli. A 900-msec blank screen delay divided the presentation and recognition probe phases of each trial.

Memory was measured using single-probe recognition, with all binding conditions involving the presentation of a unitized colorshape probe (e.g., a blue circle). Each condition contained an equal number of same and different trials, randomly intermixed. On same trials, this color-shape pairing matched one of the originally presented items, with the participants required to press the " $z$ " key on the keyboard if they thought that this feature combination had been present. On different trials, a presented color was re-paired with a shape from one of the other presented items, with the participants required to press the " $x$ " key for a correct response. Accuracy was emphasized rather than speed.

The use of a unitized probe in all the separated binding conditions was intended to explore the processes by which features are men-

\section{A Unitized Binding}

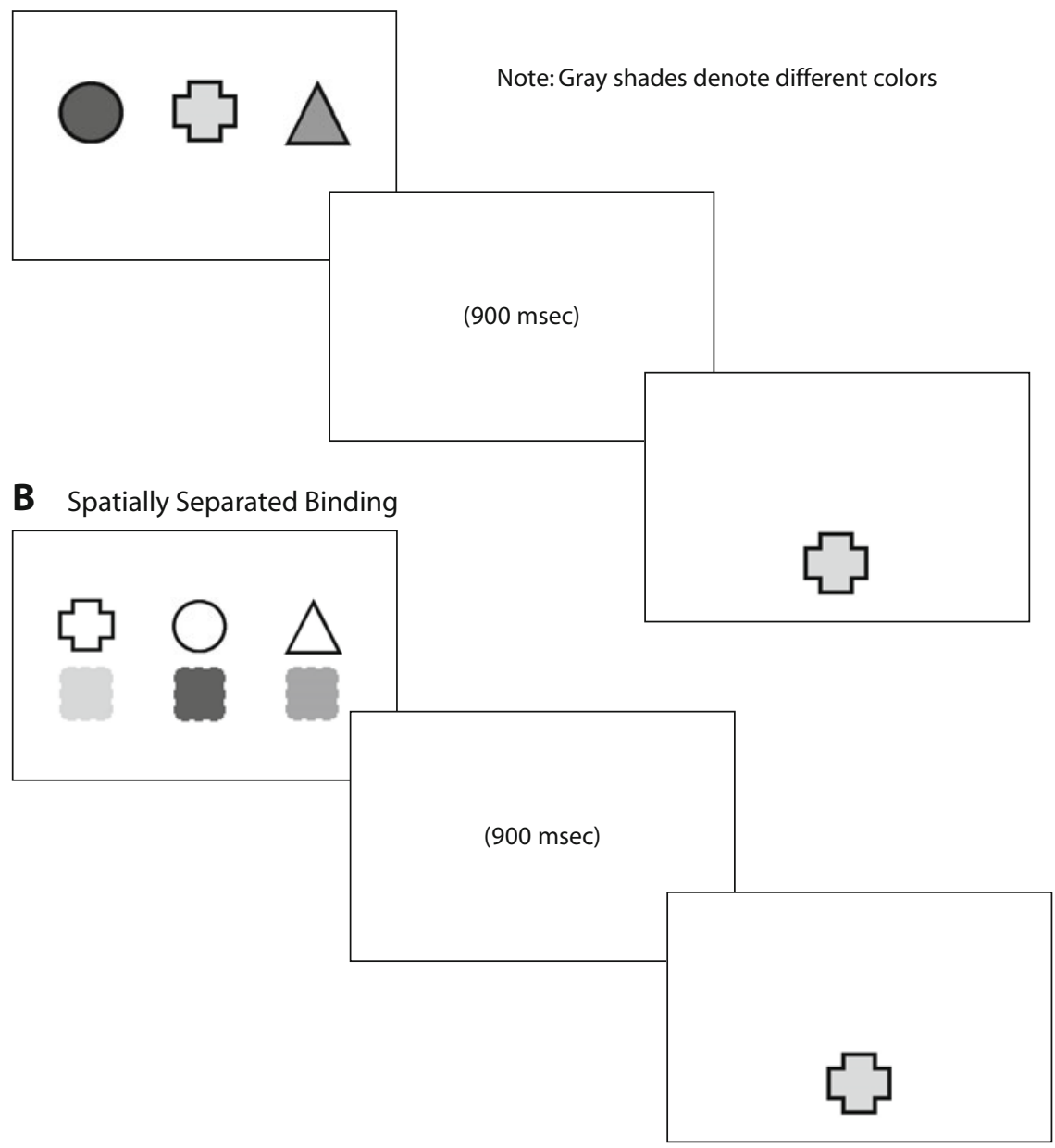

Figure 1. Illustration of stimuli and presentation procedure in unitary and spatially separated feature conjunction conditions in Experiments 3 and 4. 
tally merged, bound, or combined into single objects and compared with the test item. Recognition probes were, therefore, less similar to presented stimuli in the separated conditions than in the unitized condition. It is possible that this might contribute to overall differences in accuracy between conditions (although Allen et al., 2009, found no decrement in cross-modal binding, relative to unitized binding, using the same recognition probe procedure). This probe method may also have increased the attention demands of separated conditions. Our intention was to examine whether a significant binding condition $\times$ concurrent task interaction was present and, if so, to attempt subsequently to rule out such probe-based explanations. Evidence of equivalent executive load across binding conditions would, therefore, obviate the need to test such possible sources of the absent effect.

The demanding concurrent task used in the relevant studies was backward counting, performed during study and retention. At the beginning of each trial, a three-digit start number was presented for $2 \mathrm{sec}$ at the center of the screen. The participants were instructed to count aloud backward from this number in specified decrements (e.g., threes), until the presentation of the test probe (e.g., "Three hundred and twenty-one, three hundred and eighteen, three hundred and fifteen ....'). Such tasks have previously been shown to load on central executive resources also required in visuospatial encoding tasks (e.g., Allen et al., 2006; Allen et al., 2009; Han \& Kim, 2004). Keypress recognition responses were typically made following cessation of the counting task. Baseline measures of counting performance were also obtained, through the performance of 10 trials before and after the main body of the experiment. Counting speed was scored as the mean number of counting steps achieved on each trial.

In all the experimental conditions not involving backward counting, the participants instead performed a simple concurrent articulatory suppression task, to prevent verbal recoding and mnemonic support from the phonological loop (see Baddeley, 1986). This involved counting out loud from 1 to 4 repeatedly, at a speed of two digits per second, from the onset of each trial, through to the recognition probe.

\section{EXPERIMENTS 1-4 Spatial Separation of Features}

The first four experiments compared accuracy and fragility of unitary objects with shape-color combinations where the features were spatially separated. We expected better memory for unitized than for nonunitized objects (Delvenne \& Bruyer, 2004; Walker \& Cuthbert, 1998; $\mathrm{Xu}, 2002 \mathrm{a}, 2002 \mathrm{~b}$; but see also Allen et al., 2009). Experiments 1 and 2 used serial presentation of feature pairs, whereas Experiments 3 and 4 involved simultaneous presentation of entire arrays. In each case, the first experiment in the pair manipulated exposure duration, in order to establish appropriate presentation times and, also, to assess whether the binding of spatially separate features requires relatively longer encoding time. Experiment 1 also varied the number of items in each sequence, again to ascertain the most appropriate set size for subsequent studies. Experiments 2 and 4 then implemented a concurrent task methodology with the logic that, if spatial separation prevents the automatic encoding and retention of feature connections, disruption of general attentional resources by a demanding concurrent task should impair performance for spatially separated feature combinations. Alternatively, if unitary and spatially separated bindings place an equivalent load on central executive resources, they should be similarly affected.

\section{Experiment 1: Serial Presentation and Spatial Separation of Features}

Twenty participants (11 females and 9 males) took part in the experiment, which followed a $2 \times 2 \times 3$ repeated measures design. The three independent variables were binding type (unitary vs. spatially separated feature combinations), exposure duration ( 250 vs. $1,000 \mathrm{msec}$ ), and set size $(2,3$, or 4$)$. The binding type and exposure duration conditions were performed in counterbalanced blocks, each starting with the shortest set size and ending with the longest one. Each block contained 112 trials (32 at set size 2 and 40 each at set sizes 3 and 4), with 8 trials in each of the set size 3 and 4 blocks involving a feature repetition. Since a feature repetition across only two study items makes it impossible to recombine a feature from each item into a new lure conjunction, repetition trials were randomly distributed throughout set sizes 3 and 4 only. Each stimulus was presented at screen center for either $250 \mathrm{msec}$ or $1,000 \mathrm{msec}$, depending on the exposure duration condition, with a 250 -msec blank screen interstimulus interval separating each presentation.

Performance in each condition is reported in terms of $A^{\prime}$. The results, collapsed across set size, are displayed in Figure 2. A $2 \times 2 \times 3$ repeated measures ANOVA revealed a significant effect of binding type $[F(1,19)=$ $\left.5.64, M S_{\mathrm{e}}=.02, p<.05, \eta_{\mathrm{p}}^{2}=.23\right]$, with unitary better than separated; exposure duration $[F(1,19)=10.50$, $\left.M S_{\mathrm{e}}=.01, p<.01, \eta_{\mathrm{p}}^{2}=.36\right]$, with longer duration enhancing performance; and set size $[F(2,38)=117.95$, $\left.M S_{\mathrm{e}}=.01, p<.001, \eta_{\mathrm{p}}^{2}=.86\right]$, with level of performance decreasing with number of targets. However, none of the interactions approached significance [binding type $\times$ exposure time, $F(1,19)=1.82, M S_{\mathrm{e}}=.01, p=$ $.19, \eta_{\mathrm{p}}^{2}=.09$; binding type $\times$ set size, $F(2,38)=1.85$, $M S_{\mathrm{e}}=.003, p=.17, \eta_{\mathrm{p}}^{2}=.09$; exposure time $\times$ set size, $F(2,38)=0.01, M S_{\mathrm{e}}=.01$; binding type $\times$ exposure time $\times$ set size, $F(2,38)=1.10, M S_{\mathrm{e}}=.01, p=.34$, $\left.\eta_{\mathrm{p}}^{2}=.06\right]$.

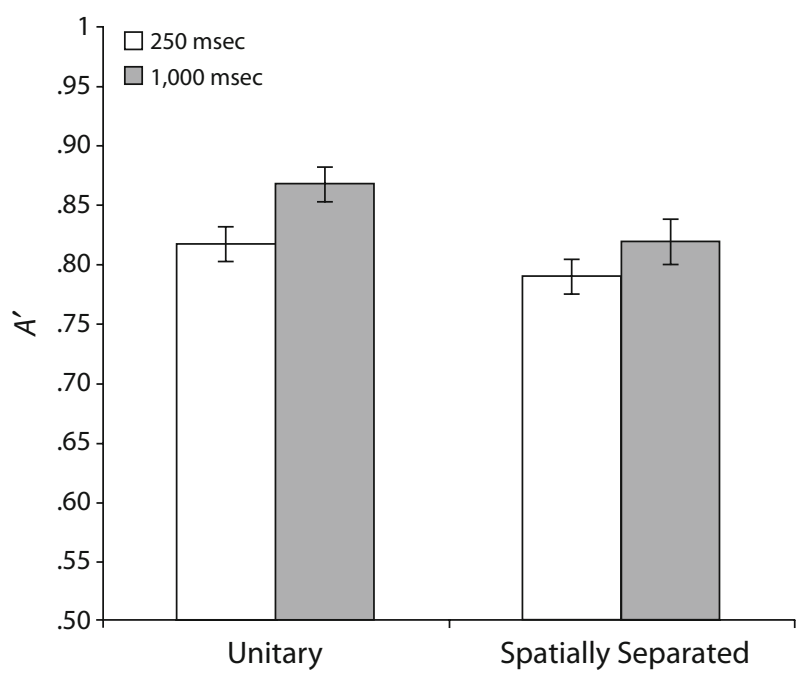

Figure 2. Accuracy $\left(A^{\prime}\right)$ (and standard errors) in Experiment 1. 
From Figure 2, it is apparent that memory was higher for unitary bindings than for spatially separated feature conjunctions, possibly reflecting the increased visual complexity of the spatially separated feature pairings. These features need to be mentally merged across space before a comparison can be made with the unitary test probe. This is likely to be error prone, causing reduced accuracy during recognition. With serial presentation, longer presentation also means a longer retention interval, thus allowing more time for short-term forgetting; yet both binding conditions benefited to the same extent from the extra study time. The absence of any interactions between binding condition and other factors suggests that the creation and maintenance of spatially separated and unitary feature conjunctions might share certain fundamental properties of processing.

\section{Experiment 2: Serial Presentation, Spatial Feature Separation, and Attentional Load}

To address whether the disparity in accuracy between binding conditions in Experiment 1 was due to differences in demands on attentional resources, the effect of a concurrent attentional load of backward counting in threes was compared with simple articulatory suppression. Twenty participants (17 females and 3 males) took part, using a $2 \times 2$ repeated measures design. Conditions were performed in four counterbalanced blocks, each containing 60 trials, $48(80 \%)$ of which were nonrepetition trials and $12(20 \%)$ of which were repetition trials, randomly distributed throughout the test block. Set size was held constant at three items and exposure duration at $1,000 \mathrm{msec}$ per item, on the basis of the performance levels observed in Experiment 1.

Accuracy in each condition is reported as $A^{\prime}$ and is displayed in Figure 3. A $2 \times 2$ repeated measures ANOVA revealed a significant effect of binding type $[F(1,19)=$ 13.83, $\left.M S_{\mathrm{e}}=.004, p<.01, \eta_{\mathrm{p}}^{2}=.42\right]$ and concurrent $\operatorname{task}\left[F(1,19)=40.47, M S_{\mathrm{e}}=.005, p<.001, \eta_{\mathrm{p}}^{2}=.68\right]$.

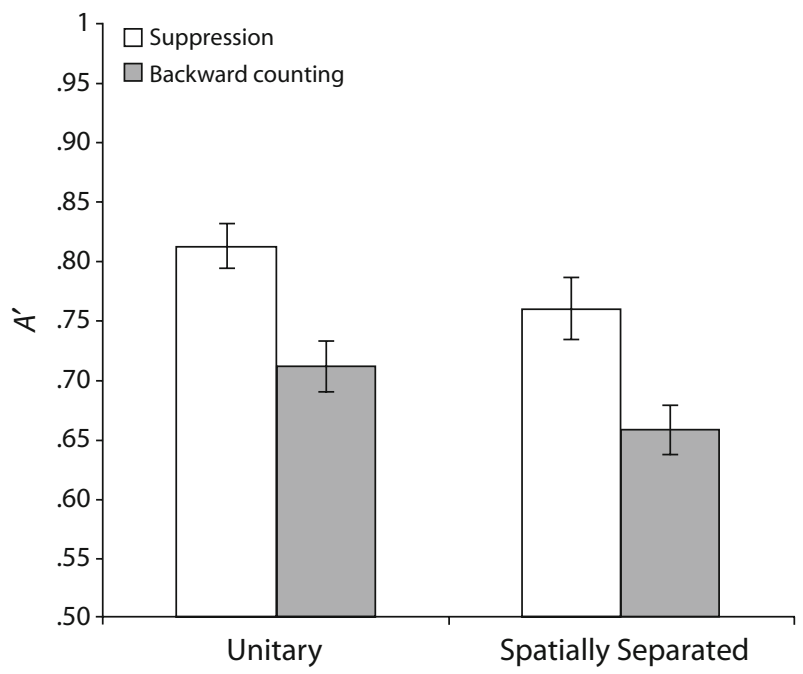

Figure 3. Accuracy $\left(A^{\prime}\right)$ (and standard errors) in Experiment 2.
However, the interaction did not approach significance $\left[F(1,19)=0.01, M S_{\mathrm{e}}=.01\right.$, n.s.], indicating that the two binding conditions were similarly affected by backward counting (Cohen's $d$, unitary bindings $=1.14$; spatially separated bindings $=0.94$ ).

Baseline and concurrent task backward counting speed was analyzed. Data from 4 participants had to be discarded because of recording problems. The remaining 16 participants scored a mean of $2.81(S E=0.21)$ counting steps on each trial with unitary bindings and a mean of $2.76(0.21)$ steps with spatially separated bindings, a nonsignificant difference $[t(15)=0.76$, n.s.; effect size $=.06]$. Backward counting, averaged across binding conditions for a mean score of $2.79(0.21)$, was slower when performed as a concurrent task than the baseline counting score of 3.17 $(0.26)[t(15)=2.61, p<.05, d=0.39]$.

The disruption of general attentional resources introduced by backward counting did not have a larger effect on spatially separated bindings. This might be surprising, since the spatially separated condition involves the initial presentation of twice as many distinct visual units. Furthermore, the requirement to judge whether a pair of features presented as a unitized recognition probe were originally encountered together requires participants to first merge or bind the separated features at encoding. Thus, although these results suggest that unitary and spatially separated features are equally susceptible to attentional interference in working memory, they do not necessarily imply common binding mechanisms.

\section{Experiment 3: Simultaneous Presentation and Spatial Feature Separation}

It is important to rule out the possibility that the absence of interactions in Experiment 2 was due to the nature of the presentation format. Accordingly, our next experiment tested binding memory using simultaneous presentation, a method that may place different demands on attention for unitized and separated binding (see Shafritz et al., 2002). When attention must be split between several competing items distributed across space, illusory bindings of features from separate items might increase, particularly when the features are also separated in space. Alternatively, unitary and spatially separated binding processes might share the same properties across presentation formats.

Twenty undergraduates (12 females and 8 males) took part in this experiment. The experiment was conducted according to a $2 \times 2$ repeated measures design, manipulating binding type (unitary vs. spatially separated feature combinations) and exposure duration (1 vs. $3 \mathrm{sec}$ ). The four conditions were performed in counterbalanced blocks, each containing 60 trials, 48 (80\%) of which were nonrepetition trials and $12(20 \%)$ of which were repetition trials. The memory task procedure is illustrated in Figure 1. Items were presented in a row subtending a visual angle of $5.60^{\circ}$ in total, with approximately $1.50^{\circ}$ between each item.

Memory accuracy in each condition $\left(A^{\prime}\right)$ is displayed in Figure 4 . A $2 \times 2$ repeated measures ANOVA revealed a significant effect of binding type $\left[F(1,19)=17.90, M S_{\mathrm{e}}=\right.$ 


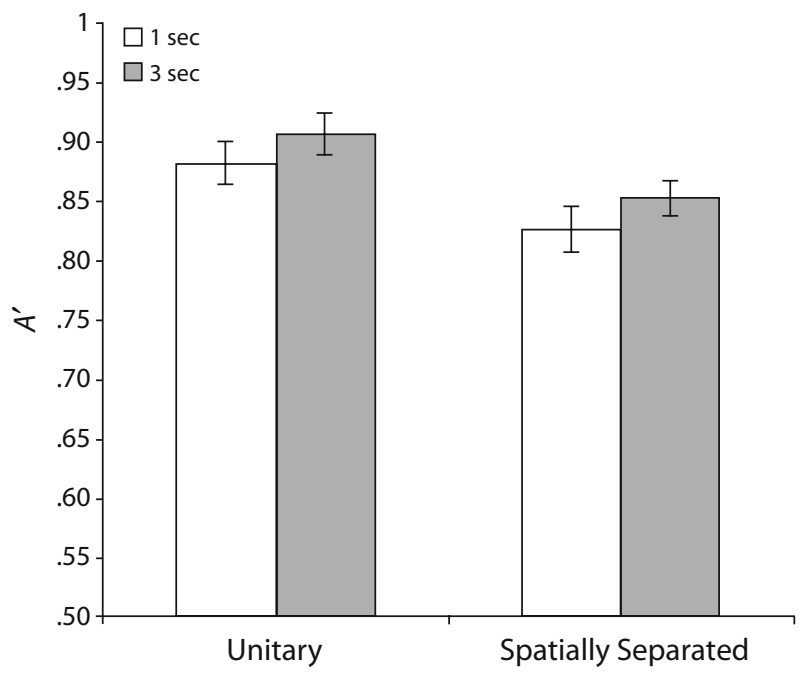

Figure 4. Accuracy $\left(A^{\prime}\right)$ (and standard errors) in Experiment 3.

$.003, p<.001, \eta_{\mathrm{p}}^{2}=.49$ ] and a marginal nonsignificant effect of exposure duration $\left[F(1,19)=4.06, M S_{\mathrm{e}}=.003\right.$, $\left.p=.058, \eta_{\mathrm{p}}^{2}=.17\right]$. The interaction did not approach significance $\left[F(1,19)=0.01, M S_{\mathrm{e}}=.002\right.$, n.s. $]$. This experiment therefore replicated the serial presentation findings in Experiment 1. Although spatially separated bindings were less well retained than unitary bindings, there was no interaction between stimulus condition and exposure duration on any of the measures. This indicates that processing and binding spatially separated features does not require a substantial degree of additional encoding time. A further experiment, involving a resource-demanding concurrent task, was performed to investigate the possible role of executive processes in binding across locations with simultaneous item presentation.

\section{Experiment 4: Simultaneous Presentation, Spatial Feature Separation, and Attentional Load}

In this experiment, we investigated the effect of executive load on memory for unitary and spatially separated feature conjunctions with simultaneous presentation. In order to ensure sensitivity to a possible binding type $\times$ task interaction, participants were discarded if they achieved a score of less than $A^{\prime}=.60$ in one or more conditions. Data are therefore reported from 20 undergraduates ( 14 females and 6 males), with data from 12 additional participants excluded. Each experimental block contained 60 trials, 48 $(80 \%)$ of which were nonrepetition trials and $12(20 \%)$ of which were repetition trials. The presentation procedure from Experiment 3 was implemented again, using a 1-sec presentation duration.

Accuracy in each condition $\left(A^{\prime}\right)$ is displayed in Figure 5. A $2 \times 2$ repeated measures ANOVA revealed a significant effect of binding type $\left[F(1,19)=9.80, M S_{\mathrm{e}}=.01, p<\right.$ $\left..01, \eta_{\mathrm{p}}^{2}=.34\right]$, as well as concurrent task $[F(1,19)=32.28$, $\left.M S_{\mathrm{e}}=.01, p<.001, \eta_{\mathrm{p}}^{2}=.63\right]$. As in Experiment 2, the interaction did not approach significance $[F(1,19)=1.64$, $\left.M S_{\mathrm{e}}=.01, p=.216, \eta_{\mathrm{p}}^{2}=.08\right]$. An analysis was also per- formed on the full set of 32 participants, again revealing effects of binding condition $\left[F(1,31)=28.80, M S_{\mathrm{e}}=.01\right.$, $\left.p<.001, \eta_{\mathrm{p}}^{2}=.48\right]$ and concurrent task $[F(1,31)=31.28$, $\left.M S_{\mathrm{e}}=.01, p<.001, \eta_{\mathrm{p}}^{2}=.50\right]$, but not the interaction $\left[F(1,31)=0.71, M S_{\mathrm{e}}=.01, \mathrm{n} . \mathrm{s}\right.$. $]$. Backward counting demands did not significantly vary with binding condition, although the effect was somewhat larger on unitary (Cohen's $d=0.97)$ than on spatially separated $(0.55)$ bindings. Therefore, the withdrawal of attentional resources had the same effect on the creation and maintenance of unitary and separated feature bindings, replicating findings from Experiment 2.

Backward-counting data from 4 participants was discarded because of problems with sound files. The remaining 16 participants scored a mean of $1.97(S E=0.15)$ counting steps with unitary bindings and a mean of 1.79 (0.13) for steps with spatially separated bindings, a significant difference $[t(15)=3.24, p<.01$, Cohen's $d$ effect size $=0.30]$. This would seem to reflect a withdrawal of attentional resources from the concurrent task toward the memory task in the spatially separated condition. The trend toward larger counting effects on unitized than on separated recognition memory would appear to indicate a trade-off between primary and secondary task performance in this experiment, since backward counting had a larger effect size on unitized, relative to separated, binding. Finally, backward counting averaged across binding conditions was slower when performed as a concurrent task [mean score $=1.88(0.14)]$ than as a single task [mean baseline score $=2.20(0.17)][t(15)=3.98, p<.01, d=0.51]$.

\section{EXPERIMENTS 5-7 Temporal Separation of Features}

Unitary and spatially separated conditions involve simultaneous presentation of color and shape. As such, although spatially separated binding may be somewhat less accurate than unitized binding, creation and subsequent

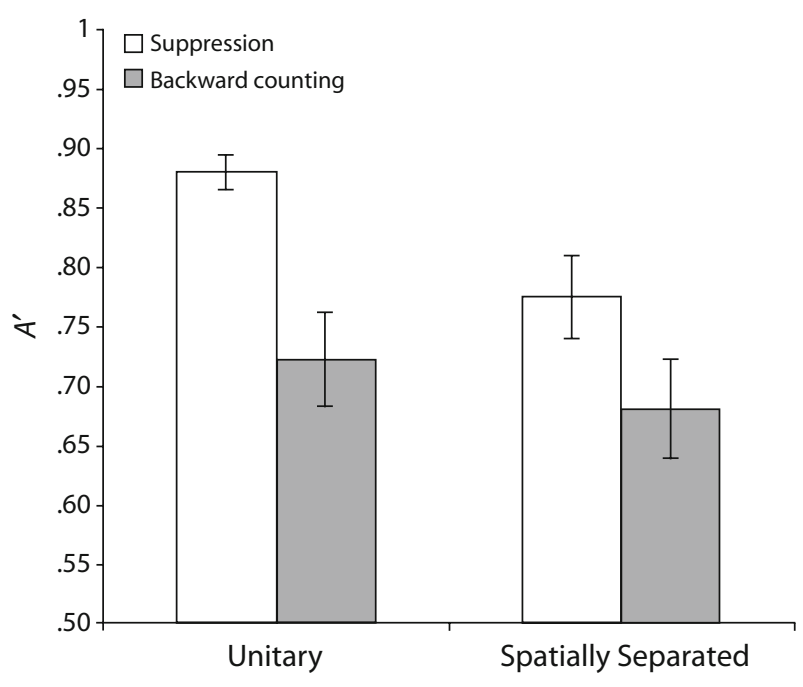

Figure 5. Accuracy $\left(A^{\prime}\right)$ (and standard errors) in Experiment 4. 
maintenance of these representations in working memory may involve relatively automatic processes. In contrast, by separating individual features in time and presenting them sequentially, the binding process may become qualitatively different and less perceptual in nature. Binding across time makes increased demands on short-term storage and, perhaps, also on the central executive. One set of features must be retained in memory while a second set of features is processed, with integration only then following. Temporally separated feature binding may therefore require additional control processes and might be particularly susceptible to decay or interference.

In Experiments 5-7, we examined accuracy in temporally separated binding, presenting shapes and colors in the same locations but separated by a brief delay. In Experiment 5, we compared this condition with memory for spatially separated feature binding, as well as memory for shapes or colors alone. In Experiments 6 and 7, we then explored the effect of executive load on unitary and temporally separated binding through the addition of demanding concurrent tasks. All of these experiments involved simultaneous presentation of three objects or features.

\section{Experiment 5: Temporal Versus Spatial Separation of Features}

Twenty undergraduates (16 females and 4 males) took part in each condition of the experiment. The independent variable was stimulus type (color only; shape only; spatially separated feature combinations; temporally separated feature combinations, color first; and temporally separated feature combinations, shape first). Since temporally separated binding requires retention of the first feature during processing of the second, it was important to compare performance when either shape or color was the first dimension to be displayed. The five conditions were performed in counterbalanced blocks, each containing 60 trials, $48(80 \%)$ of which were nonrepetition trials and $12(20 \%)$ of which were repetition trials.

As in the previous experiments, each trial began with the 2-sec delay, warning cross, and short delay. In temporally separated conditions, we then presented one of the feature dimensions (e.g., color) for $1 \mathrm{sec}$, followed by a 500 -msec delay containing a visual pattern mask (consisting of a dense black-and-white abstract pattern with an approximately $7^{\circ}$ horizontal by $4^{\circ}$ vertical visual angle) and then the other feature dimension (e.g., shape). Crucially, the two features of a pairing appeared in the same spatial location, with the participants required to remember these pairs. In color-only and shape-only conditions, all the stimuli were presented prior to the visual mask. In the spatially separated condition, the visual mask was preceded by a 1 -sec blank screen, with all relevant information being presented for $1 \mathrm{sec}$, after the mask. The recognition probe followed the standard 900-msec delay. Binding conditions used the unitary colored shape probe from previous experiments, whereas the color- and shapeonly conditions used a single feature probe (with $50 \%$ of the trials involving a feature that was not present in the display).
Table 1

Mean Accuracy $\left(A^{\prime}\right)$ and Standard Error in Each Stimulus Condition in Experiment 5

\begin{tabular}{lll}
\hline \multicolumn{1}{c}{ Condition } & $A^{\prime}$ & $S E$ \\
\hline Temporally separate (color first) & .86 & .02 \\
Temporally separate (shape first) & .75 & .03 \\
Spatially separate & .83 & .02 \\
Color only & .98 & .01 \\
Shape only & .93 & .01 \\
\hline
\end{tabular}

Mean accuracy levels $\left(A^{\prime}\right)$ for each stimulus condition are displayed in Table 1. A repeated measures ANOVA revealed a significant effect of stimulus type $[F(4,76)=$ $\left.42.40, M S_{\mathrm{e}}=.01, p<.001, \eta_{\mathrm{p}}^{2}=.69\right]$. Further comparisons revealed that accuracy was higher for color memory than for each of the binding conditions (Tukey's HSD, $p<.01$, in all cases) and was also higher than for shape memory (Tukey's HSD, $p<.05$ ). In turn, shape memory was significantly more accurate than spatially separated (Tukey's HSD, $p<.01$ ) and temporally separated (shape first) binding (Tukey's HSD, $p<.05$ ) but did not significantly differ from temporally separated (color first) binding (Tukey's HSD, $p<.05$ ). There were no differences between the three binding conditions in this analysis (Tukey's HSD, $p<.05$ ). This experiment therefore revealed equivalent accuracy for associations between features when separated either in space or in time, although both of these conditions were less accurate than simple feature memory.

We have already demonstrated that the encoding and maintenance of a set of visual objects places demands on central executive resources. The creation of associations between two sets of features, presented separately in time, might therefore be particularly dependent on attention. The final two experiments directly tested this prediction. An absence of an interaction between stimulus type and concurrent task would strongly suggest that attention is not central to the creation of bound objects in working memory, regardless of whether they are derived from unitary perceptual objects or from features encountered separately in time or space.

\section{Experiment 6: Effect of Load on Binding Temporally Separated Features}

The final two experiments compared the effect of backward counting for $2 \mathrm{sec}$ on immediate memory for temporally separated and unitary feature combinations. Eighteen undergraduates (16 females and 2 males) took part in the 65-min experiment. Data were discarded from 11 additional participants who achieved a score of less than $A^{\prime}=.60$ in one or more conditions. The experiment implemented a $3 \times 2$ repeated measures design, manipulating binding type (temporally separated bindings, early unitary bindings, or late unitary bindings) and concurrent task (simple articulatory suppression or backward counting). The six conditions were performed in counterbalanced blocks, each containing 60 trials, 48 (80\%) of which were nonrepetition trials and 12 (20\%) of which were repetition trials. 
Temporally separated binding was implemented using the same method as that in Experiment 5, although we used only the color-first version of the task. This condition was compared with two versions of unitized binding. In the early unitary binding condition, three colored shapes were presented for $1 \mathrm{sec}$ before a 500 -msec mask, at the equivalent stage to color presentation in temporally separated binding. The mask was followed by the presentation of three black dots, one in each previously occupied location, again for $1 \mathrm{sec}$. For the late unitary binding condition, the unitized binding stimuli and placeholder dots switched positions in the presentation sequence. These two versions of unitary binding were included to control for the fact that, in the temporally separate condition, one set of features had to be retained for a period of time before the other set was presented. It is important to distinguish any differential effect of attentional load due to binding conditions from that caused by variations in the duration of visual storage and processing. If binding of temporally separated features is more attention demanding, this condition should show a greater concurrent task effect than the early unitized condition (since these tasks require encoding and storage for an equivalent duration).

Accuracy $\left(A^{\prime}\right)$ in each condition is displayed in Figure 6. Concurrent task effect sizes in each condition were $d=1.84$ for early unitary binding, 1.07 for late unitary binding, and 1.60 for temporally separated binding. A $2 \times 2$ repeated measures ANOVA comparing temporally separated with early unitary binding revealed a significant effect of concurrent task $\left[F(1,17)=77.38, M S_{\mathrm{e}}=\right.$ $\left..01, p<.001, \eta_{\mathrm{p}}^{2}=.82\right]$ but no effect of stimulus condition $\left[F(1,17)=0.18, M S_{\mathrm{e}}=.01\right.$, n.s. $]$ and no stimulus condition $\times$ concurrent task interaction $[F(1,17)=0.86$, $M S_{\mathrm{e}}=.01$, n.s.]. Thus, unitary and temporally separated conditions were similarly affected by the withdrawal of attentional resources, when duration of dual-task conditions (stimulus encoding and retention + backward counting) is matched.

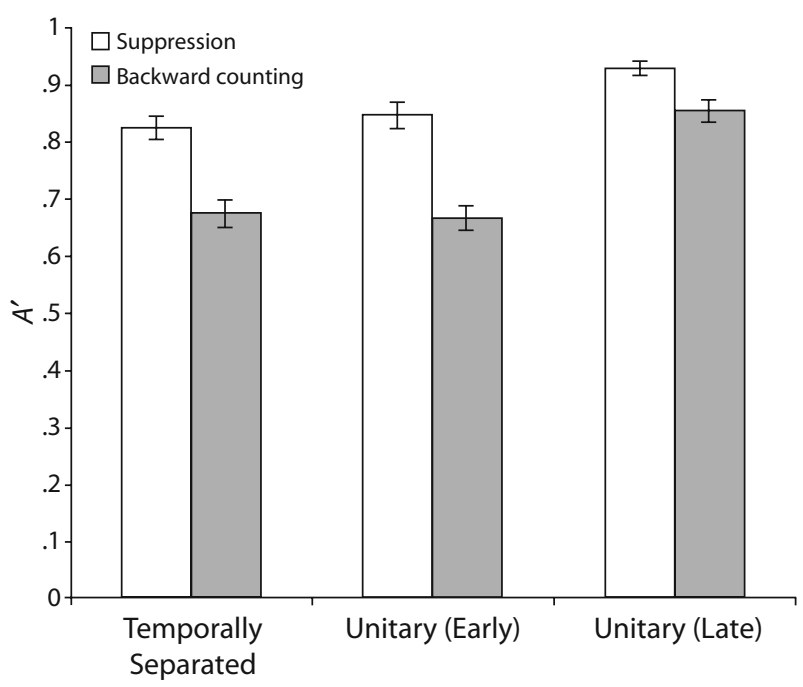

Figure 6. Accuracy $\left(A^{\prime}\right)$ (and standard errors) in Experiment 6.
A second ANOVA comparing temporally separated with late unitary binding revealed significant effects of concurrent task $\left[F(1,17)=95.74, M S_{\mathrm{e}}=.004, p<\right.$ $\left..001, \eta_{\mathrm{p}}^{2}=.85\right]$ and stimulus condition $[F(1,17)=51.45$, $\left.M S_{\mathrm{e}}=.01, p<.001, \eta_{\mathrm{p}}^{2}=.75\right]$ and a task $\times$ condition interaction $\left[F(1,17)=5.26, M S_{\mathrm{e}}=.01, p<.05, \eta_{\mathrm{p}}^{2}=\right.$ .24]. The larger effect of backward counting on temporally separated binding in this analysis presumably reflects the longer period of duration of dual-task performance in this condition. This was supported by an analysis of early and late unitary binding conditions, which also revealed significant effects of concurrent task $[F(1,17)=50.49$, $\left.M S_{\mathrm{e}}=.004, p<.001, \eta_{\mathrm{p}}^{2}=.75\right]$ and stimulus condition $\left[F(1,17)=68.80, M S_{\mathrm{e}}=.01, p<.001, \eta_{\mathrm{p}}^{2}=.80\right]$ and the task $\times$ condition interaction $\left[F(1,17)=25.00, M S_{\mathrm{e}}=.01\right.$, $\left.p<.001, \eta_{\mathrm{p}}^{2}=.60\right]$. As in Experiment 4, the same analyses were performed on all the data (including excluded participants), revealing the same pattern of findings.

Turning to backward counting, participants scored a mean of $2.62(S E=0.13)$ counting steps with early unitary bindings, a mean of $2.63(0.16)$ for late unitized, and a mean of $2.66(0.12)$ steps with temporally separated bindings. A repeated measures ANOVA revealed no significant difference in counting speed between binding conditions $\left[F(2,16)=0.21, M S_{\mathrm{e}}=4.87 \times 10^{-03}\right.$, n.s. $]$. Backward counting, averaged across binding conditions for a mean score of $2.63(0.14)$, was slower as a concurrent task $[t(17)=11.17, p<.001, d=1.89]$, relative to the baseline score of $3.83(0.16)$.

Memory for binding of temporally separated features was not significantly worse than that for visually unitized binding when stimuli were required to be retained for equivalent durations (the early unitized binding condition). This comparison will be discussed further following Experiment 7. The observation that memory for unitized stimuli was better the more recently they were presented (i.e., late vs. early) can be regarded as further evidence for the fragility of feature bindings in visual working memory (cf. Allen et al., 2006). Importantly, as with spatially separated features, the binding of features that shared spatial locations but were separated in time was not relatively more reliant on executive control than was temporally and spatially unitized binding. Although executive processes clearly contributed to memory performance, there was no evidence that they played a greater role when color had to be stored for a short interval before it could be bound with shape, relative to when color and shape were presented as unitized objects.

\section{Experiment 7: Load and Temporal Binding_A Replication}

Although the participants in Experiment 6 performed above chance in all the conditions, performance levels were somewhat low in the temporal binding condition. Therefore, a final experiment was conducted, aiming to reduce general difficulty levels. This experiment was essentially a replication of Experiment 6, with the following changes: Retention interval was reduced to $500 \mathrm{msec}$; the masked delay between feature presentation was removed; 


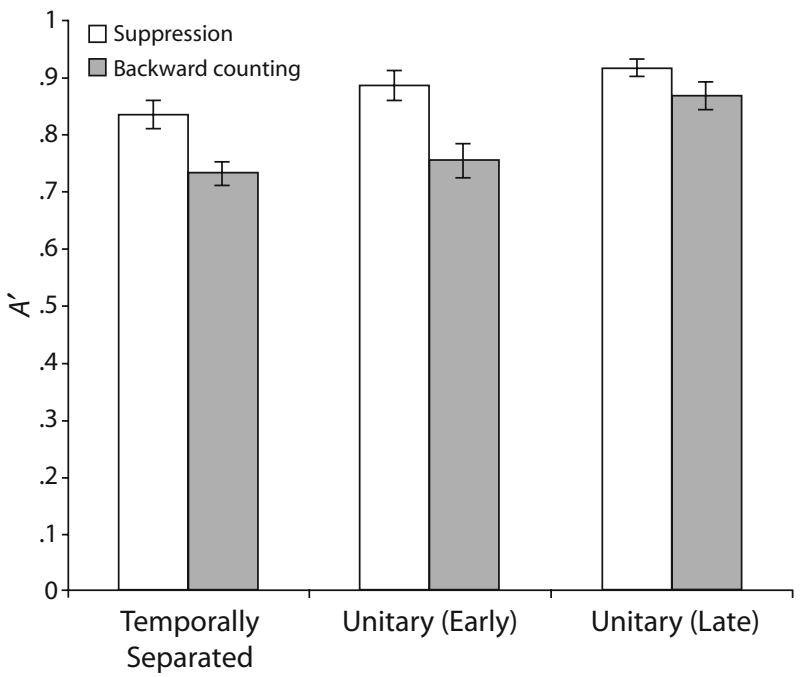

Figure 7. Accuracy $\left(A^{\prime}\right)$ (and standard errors) in Experiment 7.

and instead of dots, a blank screen was presented before or after the unitary bindings. In addition, there were fewer trials, and the participants controlled the pace of the experiment by triggering the start of each trial by pressing the space bar.

Eighteen undergraduates ( 14 females and 4 males) took part in the 60-min experiment, whereas data from 7 additional participants were discarded since they achieved a score of less than $A^{\prime}=.60$ in one or more conditions. The six conditions were performed in counterbalanced blocks, each containing 48 trials, 36 (75\%) of which were nonrepetition trials and $12(25 \%)$ of which were repetition trials.

Accuracy $\left(A^{\prime}\right)$ is displayed in Figure 7. Concurrent task effect sizes in each condition were $d=1.11$ for early unitary binding, 0.46 for late unitary binding, and 1.07 for temporally separated binding. A $2 \times 2$ ANOVA comparing temporally separated bindings with early unitized bindings revealed significant effects of binding type $\left[F(1,17)=4.57, M S_{\mathrm{e}}=.01, p<.05, \eta_{\mathrm{p}}^{2}=.21\right]$ and concurrent task $\left[F(1,17)=40.12, M S_{\mathrm{e}}=.01, p<.001, \eta_{\mathrm{p}}^{2}=\right.$ .70] but no significant interaction $\left[F(1,17)=0.40, M S_{\mathrm{e}}=\right.$ .01 , n.s.]. Similarly, a comparison of temporally separated bindings and late unitized bindings also revealed significant effects of binding type $\left[F(1,17)=33.52, M S_{\mathrm{e}}=.01\right.$, $\left.p<.001, \eta_{\mathrm{p}}^{2}=.66\right]$ and concurrent task $[F(1,17)=56.91$, $\left.M S_{\mathrm{e}}=.002, p<.001, \eta_{\mathrm{p}}^{2}=.77\right]$ but no significant task $\times$ condition interaction $\left[F(1,17)=2.48, M S_{\mathrm{e}}=.01, p=\right.$ $\left..133, \eta_{\mathrm{p}}^{2}=.13\right]$. As in Experiment 6, a comparison of early and late unitary binding conditions revealed significant effects of concurrent task $\left[F(1,17)=41.14, M S_{\mathrm{e}}=.004\right.$, $\left.p<.001, \eta_{\mathrm{p}}^{2}=.71\right]$ and stimulus condition $[F(1,17)=$ 21.26, $\left.M S_{\mathrm{e}}=.004, p<.001, \eta_{\mathrm{p}}^{2}=.56\right]$ and a significant task $\times$ condition interaction $\left[F(1,17)=6.23, M S_{\mathrm{e}}=.01\right.$, $\left.p<.05, \eta_{\mathrm{p}}^{2}=.27\right]$. Finally, analyzing data from all the participants (including those excluded from the analyses above) produced the same pattern of findings. Overall, even when particular care was taken to avoid floor effects, the binding of unitary features and that of temporally separated features were similarly affected by the withdrawal of attentional resources when processing and storage duration was controlled.

The participants achieved a mean backward counting score of $2.21(S E=0.13)$ counting steps with early unitary bindings, $2.25(0.12)$ with late unitary bindings, and $2.10(0.11)$ with temporally separated bindings. A repeated measures ANOVA revealed no significant difference in counting speed between the three binding conditions $\left[F(2,16)=2.13, M S_{\mathrm{e}}=5.41 \times 10^{-02}\right.$, n.s. $]$. Backward counting, averaged across binding conditions for a mean score of $2.23(0.12)$, was again slower as a concurrent task $[t(17)=3.23, p<.01, d=0.37]$, relative to the baseline score of $2.39(0.13)$.

In conclusion, changing the procedure used in Experiment 6 had only a marginal effect on overall levels of performance and reduced only slightly the number of participants excluded for failing to exceed chance performance. Although the pattern of results was much the same as before, unitized colored shapes were remembered better whether presented early or late in the sequence, relative to temporally separated presentation of colors and then shapes (in contrast to Experiment 6). We assume that the changes to the experimental procedure were successful in making the experiment more sensitive to our manipulation of binding. Despite this, however, there was again no evidence of a relatively higher executive load in the temporally separated binding condition. Counting backward disrupted memory for feature combinations equally for the temporally separated and visually unitized conditions, providing further evidence that although binding features across time places extra demands on working memory, it does not result in a higher load on the central executive.

\section{DISCUSSION}

It is assumed that features are bound into chunks in memory when the retrieval of one component is sufficient to evoke the remainder. It seems reasonable to assume that there are many different types of binding, depending on what subsystems of working memory and longterm memory are involved. In order to develop a comprehensive theory of binding, it is necessary to identify and delineate when feature integration occurs automatically and when more resource-demanding binding processes are invoked. The degree to which bindings are retained automatically or depend on additional central resources may be the result of a combination of factors, including object complexity and configuration, exposure duration, and retention interval.

Luck and Vogel (1997; Vogel et al., 2001) argued that simple visual features are automatically bound and retained as integrated objects in working memory, possibly through a mechanism such as synchronous neural firing. Allen et al. (2006) found that, when attentional resources were disrupted using the dual-task methodology, conjunction memory was no more impaired than memory for single features. The memory advantage gained from Gestalt 
factors such as continuation and symmetry also appears to be relatively cost free in terms of attentional resources, with demanding concurrent activity again failing to cause differential disruption (Rossi-Arnaud et al., 2006; Woodman et al., 2003). It seems plausible to assume that these binding processes operate at a relatively peripheral perceptual level, before the resultant binding is registered in working memory.

Our present study was an attempt to study binding under potentially more demanding conditions in which the respective color and shape features were separated in space and time. During study and a brief retention interval, participants performed a concurrent counting task assumed to tap the central executive, while at the same time placing minimal demands on visuospatial processing. We observed that this withdrawal of attentional resources had no additional effect when to-be-bound features were separated, rather than presented as a unitary object. Although Experiments 1 and 3 showed reduced recognition accuracy with spatial separation, both with sequential and simultaneous presentation modes, Experiments 2 and 4 indicated that this decline was not due to the disruption of general attentional resources. Although temporal separation of features also reduced recognition accuracy, Experiments 6 and 7 suggested that this reduction, too, was not due to added competition for attentional resources. Although recognition accuracy varied across experiments with manipulation of different elements of the task, no significant stimulus condition $\times$ concurrent task interaction was observed.

Before discussing these findings further, it is important to acknowledge the difficulty of drawing firm conclusions from what are essentially null interaction results. It is unlikely, however, that our failure to observe a significant interaction between binding condition and concurrent task was due to a lack of power. If accuracy levels in the separated conditions were relatively more reliant on the degree of attentional support available, they should show a greater effect of backward counting, or at least a trend toward this. However, examination of the concurrent task effect sizes in each experiment actually reveals a consistent trend toward slightly larger effects on unitized than on separated binding conditions. The only evidence that emerged for a greater attentional load in the separated conditions was a slower backward counting rate in Experiment 4 , and this was likely the result of a differential trade-off pattern between primary and secondary tasks in this study. The consistent absence of a larger task effect on separated binding across several experiments means that we have little evidence for a particular load on attention in these conditions.

\section{Spatially and Temporally Separated Binding}

The obvious interpretation of these findings is to suggest that the binding of spatially or temporally separated features is automatic. However, a strong form of this claim would appear to be less than plausible. A perceptual system that always automatically bound colors and shapes that were not colocated as part of the same object, or bound features that were separated in time, would surely lead to perceptual chaos. Furthermore, the fact that some participants had to be excluded because of chance performance in one or more condition indicates that performance under these conditions is far from automatic. Nevertheless, spatially and temporally separated feature pairings were able to be stored in memory and did not show a relatively larger effect of concurrent executive load.

It may be that, although the binding of separated features does not critically rely on executive attention, it also does not emerge automatically through perceptual processes in the same manner as unitized binding. Participants may be able to strategically bind together separated features in response to the demands of the task, through processes that do not place any additional demands on executive support. Future experimental work could explore this possible distinction between automatic, perceptual binding and strategic but relatively cost-free binding of separated features.

A further explanation might be that a degree of association can be created between features that are colocated or at least proximal in space, even when they are not visually unitized. This process could be critically dependent on relative feature configuration and would yield a lower level of accuracy than would unitized features in subsequent recognition, consistent with the reported data. Effectively, this explanation involves adopting a relatively fuzzy account of what constitutes an object, for which, at present, there is relatively little evidence. The concept of an object would be stretched further by the observation that concurrent load does not interact with binding, even when one feature is presented visually and the other auditorily (Allen et al., 2009). Finally, even if one accepts this extended concept, we are still left with the question of how the binding process operates and the conclusion that it does not depend on general attentional capacity.

Another alternative explanation is that the spatially and temporally separated conditions do not require any form of binding during encoding or maintenance and that each of the features are, instead, retained independently in working memory. According to this argument, the recognition test could be performed by deciding whether the features of the test probe were spatially or temporally proximal during presentation. However, this would still require each of the six individual features to be bound to their locations at encoding. In addition, if distinct unbound features are stored as separate objects in working memory and its capacity is object limited (e.g., Luck \& Vogel, 1997), performance in the separated condition should be much lower than that in the unitized condition, since this would mean a load of six, as opposed to three, chunks. Instead, we observed that separated binding accuracy was only slightly lower than unitized binding performance. Thus, we can argue that features within the separated conditions were bound together in some form during encoding and maintenance. Although the data do not allow a specification of the precise nature of this binding and the underlying representations, it is emerging that the creation of bindings is relatively cost free in terms of attention even when 
features are not encountered as part of the same spatially and temporally unitized object, although these representations are also fragile and liable to disintegrate (Allen et al., 2006).

\section{Binding and the Episodic Buffer}

In our own case, this line of research was prompted by the concept of an episodic buffer, a multidimensional temporary storage system that was responsible for binding features across perceptual dimensions and between working and long-term memory. In its original form (Baddeley, 2000 ), the system was assumed to depend crucially on the operation of the central executive. For reasons of parsimony, no direct link with either the visuospatial sketchpad or the phonological loop was assumed, the reason being that this assumption could be tested directly by studying the effect of disrupting the executive on subsequent binding.

The concept of binding is itself open to interpretation. As was outlined earlier, a potentially useful distinction might be made between automatic and effortful (attentiondemanding) binding. Examples of automatic binding might be the combination of shape and color into an object or, indeed, the binding in episodic memory of an incident and the location at which it occurred. Active, effortful binding might apply to situations in which attentional manipulation is required - for example, forming a novel interactive mental image, such as that of a camel juggling, or perhaps combining separated elements into bound objects through creative visualization (see Hsi, Linn, \& Bell, 1997; Pearson, Logie, \& Gilhooly, 1999).

We assumed that our present study would reflect this more active binding, at the same time as using material comparable to that in our earlier studies of relatively automatic binding. In fact, we find no difference, other than in overall performance level. Such a result is comparable to our parallel finding from the binding of words into sentences, which again appears to be no more disrupted by concurrent activity than is the retention of the individual words (e.g., Allen \& Baddeley, 2008; Baddeley, Hitch, \& Allen, 2009). This leads to a possible revision of our view of the episodic buffer, regarding it as a system for passively storing chunks of information, rather than a system that itself actively binds.

What might set the limit of such a system, if not executive control? One possibility is that storage capacity of the buffer is limited to about four chunks, with a chunk comprising features that are integrated in such a way as to allow the retrieval of one to evoke the remainder. For chunking to occur, there needs to be some form of support, which, in the case of memory for sentences, presumably comes from semantic and syntactic language habits and, in the case of retention of objects, from other perceptual sources such as colocation, symmetry, or continuity. In some cases, these benefits are likely to occur automatically, although the results may be relatively fragile and susceptible to interference. In others, executive processing may be needed to bring together the features into chunks, but in each case, the actual storage within the episodic buffer might be assumed to be passive. Although the precise nature of the binding processes operating in the reported experiments remains to be understood, our findings nevertheless provide clear and somewhat surprising evidence that visual features separated over spatial or temporal distance can be associated in working memory without requiring additional executive control.

\section{AUTHOR NOTE}

This work was supported by Grant 160675/V50 from the Norwegian Research Council. The authors gratefully acknowledge the assistance of Anders Gravir Imenes and Øystein Sjåstad in testing the participants. Correspondence concerning this article should be addressed to R. J. Allen, Institute of Psychological Sciences, University of Leeds, Leeds LS2 9JT, England (e-mail: r.allen@1eeds.ac.uk).

\section{REFERENCES}

Allen, R. J., \& BAdDeley, A. D. (2008). Working memory and sentence recall. In A. Thorn \& M. Page (Eds.), Interactions between short-term and long-term memory in the verbal domain (pp. 63-85). Hove, U.K.: Psychology Press.

Allen, R. J., Baddeley, A. D., \& Hitch, G. J. (2006). Is the binding of visual features in working memory resource-demanding? Journal of Experimental Psychology: General, 135, 298-313.

Allen, R. J., Hitch, G. J., \& Baddeley, A. D. (2009). Cross-modal binding and working memory. Visual Cognition, 17, 83-102.

Alvarez, G. A., \& Thompson, T. W. (2009). Overwriting and rebinding: Why feature-switch detection tasks underestimate the binding capacity of visual working memory. Visual Cognition, 17, 141-159.

Asch, S. E., Ceraso, J., \& Heimer, W. (1960). Perceptual conditions of association. Psychological Monographs: General \& Applied, 74(3, Whole No. 490), 1-48.

BAdDeley, A. D. (1986). Working memory. Oxford: Oxford University Press, Clarendon Press.

BadDeley, A. D. (1996). Exploring the central executive. Quarterly Journal of Experimental Psychology, 49A, 5-28.

BAdDEley, A. D. (2000). The episodic buffer: A new component of working memory? Trends in Cognitive Sciences, 4, 417-423.

Baddeley, A. D. (2002). Fractionating the central executive. In D. T. Stuss \& R. T. Knight (Eds.), Principles of frontal lobe function (pp. 246-260). New York: Oxford University Press.

BADDELEY, A. D. (2007). Working memory, thought, and action. Oxford: Oxford University Press.

Baddeley, A. D., \& Hitch, G. J. (1974). Working memory. In G. H. Bower (Ed.), The psychology of learning and motivation: Advances in research and theory (Vol. 8, pp. 47-90). New York: Academic Press.

Baddeley, A. D., Hitch, G. J., \& Allen, R. J. (2009). Working memory and binding in sentence recall. Journal of Memory \& Language, 61, 438-456.

Boström, C. G., \& Kugelberg, I. (1981). Tabula Pseudoisochromaticae B.-K. (3rd ed.). Stockholm, Sweden: Nordiska Bokhandelns Förlag.

Cowan, N., Naveh-Benjamin, M., Kilb, A., \& Saults, J. S. (2006). Life-span development of visual working memory: When is feature binding difficult? Developmental Psychology, 42, 1089-1102.

Delvenne, J.-F., \& BruYer, R. (2004). Does visual short-term memory store bound features? Visual Cognition, 11, 1-27.

Fougnie, D., \& MARoIs, R. (2009). Attentive tracking disrupts feature binding in visual working memory. Visual Cognition, 17, 48-66.

Gajewski, D. A., \& Brockmole, J. R. (2006). Feature bindings endure without attention: Evidence from an explicit recall task. Psychonomic Bulletin \& Review, 13, 581-587.

HAN, S.-H., \& KIM, M.-S. (2004). Visual search does not remain efficient when executive working memory is working. Psychological Science, 15, 623-628.

Holcombe, A. O., \& Cavanagh, P. (2001). Early binding of feature pairs for visual perception. Nature Neuroscience, 4, 127-128.

Hsi, S., LinN, M. C., \& BeLl, J. A. (1997). The role of spatial reasoning in engineering and the design of spatial instructions. Journal of Engineering Education, 86, 151-158. 
Johnson, J. S., Hollingworth, A., \& Luck, S. J. (2008). The role of attention in the maintenance of feature bindings in visual short-term memory. Journal of Experimental Psychology: Human Perception \& Performance, 34, 41-55.

Kane, M. J., \& Engle, R. W. (2002). The role of prefrontal cortex in working-memory capacity, executive attention, and general fluid intelligence: An individual-differences perspective. Psychonomic Bulletin \& Review, 9, 637-671.

Logie, R. H., Brockmole, J. R., \& Vandenbroucke, A. R. E. (2009). Bound feature combinations in visual short-term memory are fragile but influence long-term learning. Visual Cognition, 17, 160-179.

LUCK, S. J., \& VoGEL, E. K. (1997). The capacity of visual working memory for features and conjunctions. Nature, 390, 279-281.

Miyake, A., Friedman, N. P., Emerson, M. J., Witzki, A. H., HowERTER, A., \& WAGER, T. D. (2000). The unity and diversity of executive functions and their contributions to complex "frontal lobe" tasks: A latent variable analysis. Cognitive Psychology, 41, 49-100.

Miyake, A., Friedman, N. P., Rettinger, D. A., Shah, P., \& Hegarty, M. (2001). How are visuospatial working memory, executive functioning, and spatial abilities related? A latent-variable analysis. Journal of Experimental Psychology: General, 130, 621-640.

Pearson, D. G., Logie, R. H., \& Gilhooly, K. J. (1999). Verbal representations and spatial manipulation during mental synthesis. European Journal of Cognitive Psychology, 11, 295-314.

Posner, M. I., Inhoff, A. W., Friedrich, F. J., \& Cohen, A. (1987). Isolating attentional systems: A cognitive-anatomical analysis. Psychobiology, 15, 107-121.

Rossi-Arnaud, C., Pieroni, L., \& Baddeley, A. D. (2006). Symmetry and binding in visuo-spatial working memory. Neuroscience, 139, 393-400.

Shafritz, K. M., Gore, J. C., \& Marois, R. (2002). The role of the parietal cortex in visual feature binding. Proceedings of the National Academy of Sciences, 99, 10917-10922.
Stuss, D. T., \& Knight, R. T. (EDs.) (2002). Principles of frontal lobe function. New York: Oxford University Press.

Treisman, A. [M.] (1982). Perceptual grouping and attention in visual search for features and for objects. Journal of Experimental Psychology: Human Perception \& Performance, 8, 194-214.

Treisman, A. M., \& Gelade, G. (1980). A feature-integration theory of attention. Cognitive Psychology, 12, 97-136.

Vogel, E. K., Woodman, G. F., \& Luck, S. J. (2001). Storage of features, conjunctions, and objects in visual working memory. Journal of Experimental Psychology: Human Perception \& Performance, 27, 92-114.

WALKeR, P., \& CUTHBeRT, L. (1998). Remembering visual feature conjunctions: Visual memory for shape-colour associations is objectbased. Visual Cognition, 5, 409-455.

Wheeler, M. E., \& Treisman, A. M. (2002). Binding in short-term visual memory. Journal of Experimental Psychology: General, 131, 48-64.

Woodman, G. F., Vecera, S. P., \& Luck, S. J. (2003). Perceptual organization influences visual working memory. Psychonomic Bulletin \& Review, 10, 80-87.

Xu, Y. (2002a). Encoding color and shape from different parts of an object in visual short-term memory. Perception \& Psychophysics, 64, 1260-1280.

Xu, Y. (2002b). Limitations of object-based feature encoding in visual short-term memory. Journal of Experimental Psychology: Human Perception \& Performance, 28, 458-468.

YeH, Y.-Y., YANG, C.-T., \& CHIU, Y.-C. (2005). Binding or prioritization: The role of selective attention in visual short-term memory. Visual Cognition, 12, 759-799.

(Manuscript received February 27, 2009; revision accepted for publication October 5, 2009.) 